\title{
Cytochrome P450-mediated hydroxylation is required for polyketide macrolactonization in stambomycin biosynthesis
}

\author{
Lijiang Song ${ }^{1}$, Luisa Laureti ${ }^{2,3,4}$, Christophe Corre ${ }^{1}$, Pierre Leblond ${ }^{2,3}$, Bertrand Aigle $^{2,3}$ and \\ Gregory L Challis ${ }^{1}$
}

Many polyketide antibiotics contain macrolactones that arise from polyketide synthase chain release via thioesterase (TE) domain-catalyzed macrolactonization. The hydroxyl groups utilized in such macrolactonization reactions typically derive from reduction of $\beta$-ketothioester intermediates in polyketide chain assembly. The stambomycins are a group of novel macrolide antibiotics with promising anticancer activity that we recently discovered via rational activation of a silent polyketide biosynthetic gene cluster in Streptomyces ambofaciens. Here we report that the hydroxyl group utilized for formation of the macrolactone in the stambomycins is derived from cytochrome P450-catalyzed hydroxylation of the polyketide chain rather than keto reduction during chain assembly. This is a novel mechanism for macrolactone formation in polyketide antibiotic biosynthesis.

The Journal of Antibiotics (2014) 67, 71-76; doi:10.1038/ja.2013.119; published online 13 November 2013

Keywords: acyl carrier protein; cytochrome P450; hydroxylation; macrolactonization; polyketide synthase; Streptomyces ambofaciens; thioesterase

\section{INTRODUCTION}

Several polyketide natural products with important applications in medicine and agriculture contain a macrolactone. Examples include the antibacterial erythromycin $\mathrm{A},{ }^{1}$ the antifungals amphotericin $\mathrm{B}$ and nystatin, ${ }^{2,3}$ the insecticide spinosyn $\mathrm{A}^{4}$ and the avermectins ${ }^{5}$ that are used as both insecticides and anthelmintics. Such macrolactones are typically biosynthesized by thioesterase (TE) domains located at the C-terminus of type I modular polyketide synthase (PKS) assembly lines. ${ }^{6-10}$ The fully assembled polyketide chain is transferred from the acyl carrier protein domain within the last module of the PKS to a conserved Ser residue in the active site of the TE domain. ${ }^{11}$ The resulting ester undergoes general base-assisted intramolecular nucleophilic attack by a hydroxyl group, generated by reduction of a $\beta$-ketothioester intermediate in polyketide chain assembly. ${ }^{11}$ This leads to formation of a tetrahedral intermediate that collapses to release the product of the PKS as a macrolactone (Figure 1). ${ }^{11}$

We recently reported the discovery of the stambomycins 1-4, a remarkable family of 51-membered macrolide antibiotics with promising activity against human cancer cell lines, via rational activation of a silent gene cluster in Streptomyces ambofaciens ATCC23877 that encodes a cryptic modular PKS assembly line (Figure 2). ${ }^{12}$ Although the stambomycin PKS contains a C-terminal TE domain that is proposed to catalyze macrolactone formation, ${ }^{12}$ the C-50 hydroxyl group utilized for this process does not appear to derive from PKSmediated keto reduction during polyketide chain assembly. Instead, we hypothesized that this hydroxyl group and the C-28 hydroxyl group are derived from molecular oxygen via cytochrome $P 450$ (CYP)-catalyzed hydroxylation reactions (Figure 2). ${ }^{12}$ Here we report the results of experiments aimed at investigating this hypothesis.

\section{MATERIALS AND METHODS}

HR MS, MS/MS and UHPLC-MS were carried out using a Bruker MaXis electrospray ionization time-of-flight (ESI-TOF) MS connected, where appropriate, to a Dionex 3000 RS UHPLC fitted with an Agilent Zorbax Eclipse Plus (Agilent, Wokingham, UK) C18 column $(100 \times 2.1 \mathrm{~mm}, 1.8 \mu \mathrm{m})$. NMR spectra were recorded at $25^{\circ} \mathrm{C}$ in $\mathrm{CD}_{3} \mathrm{OD}$ on a Bruker Avance II $700 \mathrm{MHz}$ spectrometer (Bruker, Coventry, UK) equipped with a TCI cryoprobe. Stambomycin-related metabolites accumulated in S. ambofaciens mutants were purified by semi-preparative HPLC on an Agilent 1100 instrument fitted with a C-18 column $(100 \times 21 \mathrm{~mm}, 5 \mu \mathrm{m}$, Agilent). The strains and plasmids used in this study are listed in Table 1 .

Deletion of samR0478 and samR0479 in S. ambofaciens The samR0478 and samR0479 genes in S. ambofaciens ATCC/OE484 ${ }^{12}$ were replaced with a spectinomycin resistance cassette using the REDIRECT system,

\footnotetext{
${ }^{1}$ Department of Chemistry, University of Warwick, Coventry, UK; ${ }^{2}$ Université de Lorraine, Dynamique des Génomes et Adaptation Microbienne, UMR 1128, Vandoeuvre-lèsNancy, France and ${ }^{3}$ INRA, Dynamique des Génomes et Adaptation Microbienne, UMR 1128, Vandoeuvre-lès-Nancy, France

${ }^{4}$ Current address: Cancer Research Center of Marseille, CNRS UMR7258, Marseille, France.

Correspondence: Professor GL Challis, Department of Chemistry, University of Warwick, Gibbett Hill Road, Coventry CV4 7AL, UK.

E-mail: g.l.challis@warwick.ac.uk

Received 22 July 2013; revised 14 October 2013; accepted 17 October 2013; published online 13 November 2013
} 


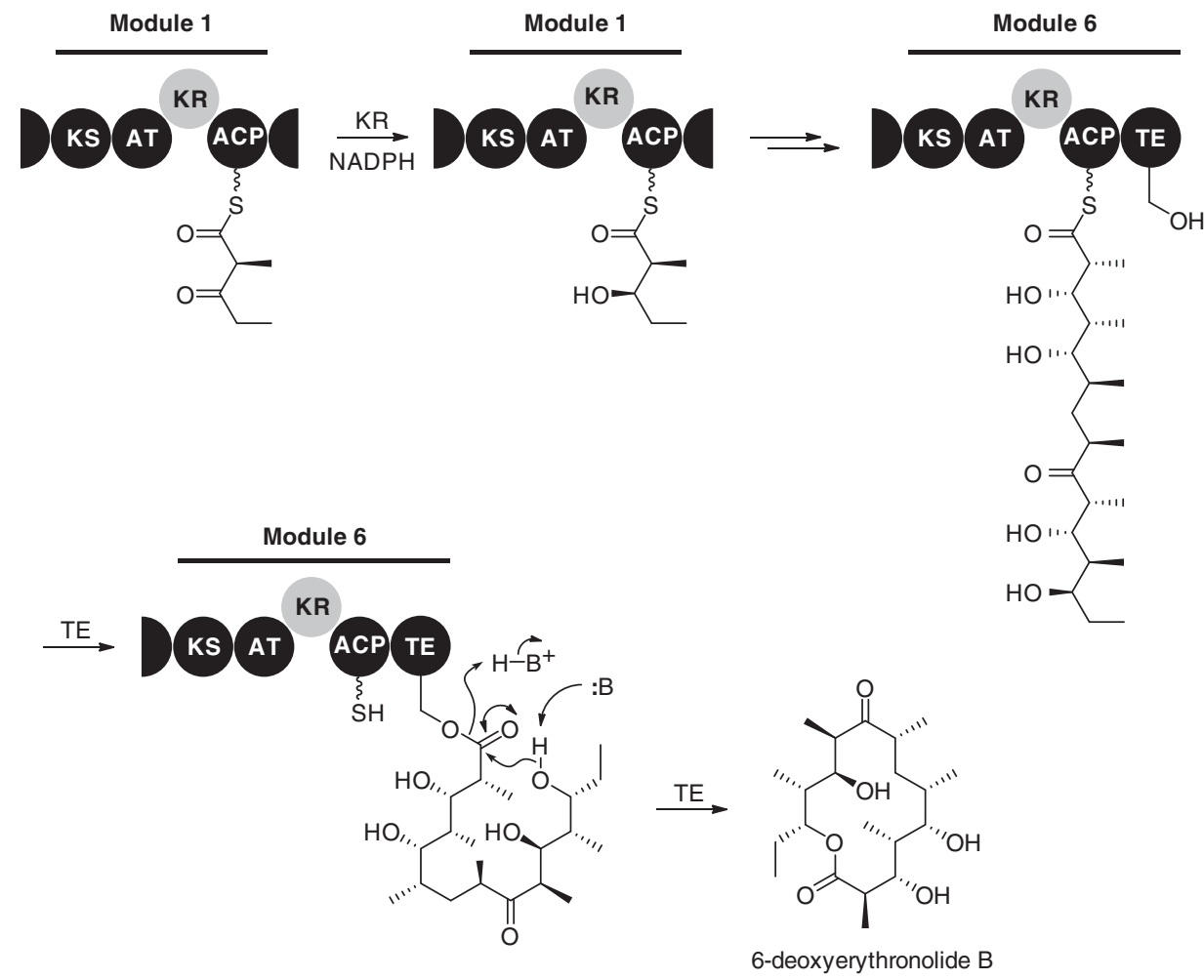

Figure 1 Mechanism for polyketide chain release from type I modular polyketide synthases (PKSs) via macrolactonization. The hydroxyl group required for macrolactonization is typically generated by reduction of a $\beta$-ketothioester intermediate in polyketide chain assembly; in this example, the ketoreductase (KR) domain within module 1 of 6 -deoxyerythronolide B synthase catalyzes this reaction. The fully assembled polyketide chain is transferred from the phosphopantetheine thiol of the acyl carrier protein (ACP) domain within the last module of the PKS to the active site serine residue of the thioesterase (TE) domain at the C-terminus of the last module. The TE domain then utilizes an active site His residue (B) as a general base to form the macrolactone (in this case 6-deoxyerythronolide B) via an addition-elimination mechanism.

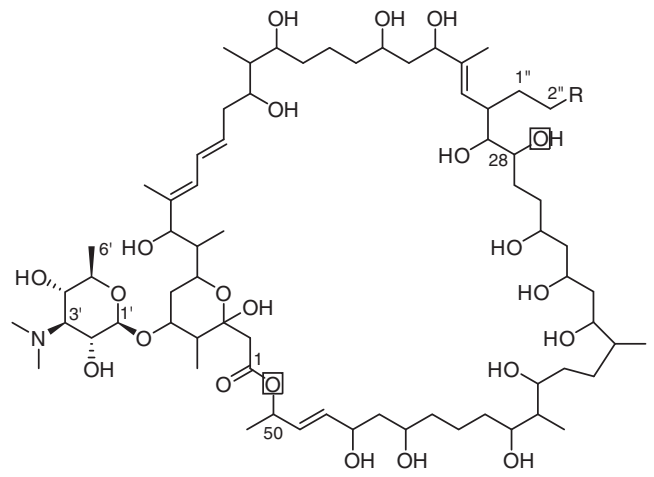

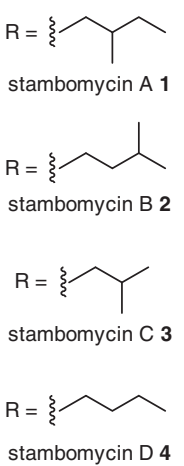

Figure 2 Chemical structures of stambomycins A-D 1-4. The two oxygen atoms proposed to derive from cytochrome P450-catalyzed hydroxylation of the polyketide chain rather than keto reduction during polyketide chain assembly are boxed.

as described previously. ${ }^{13}$ The spectinomycin resistance cassette, derived from the plasmid pIJ778, ${ }^{13}$ was used as the template for PCR reactions with the primers 5 -TGCAGACGGGCTGCTCGACGCGCGGGGAGTGACGGCATGTG TAGGCTGGAGCTGCTTC-3' (forward)/5'-GTCCCGGGCTCGGGCCGCGCC TTTGCCGCTGCCGGACTCATTCCGGGGATCCGTCGACC-3' (reverse) and 5'-GAGCGGCCGGGAGGCGGGCGCGCGGCGCAGTCGGCGTTCTGTAGGC TGGAGCTGCTTC-3' (forward)/5'-CGCCCCGCAGGACACGGCAACCACCG ACGTAGAGGGATGATTCCGGGGATCCGTCGACC- $3^{\prime}$ (reverse) to create disruption cassettes $(\sim 1400 \mathrm{bp})$ for samR0478 and samR0479, respectively.
Escherichia coli BW25113/pKD20 was transformed with the bacterial artificial chromosome (BAC) BBC and then separately with each of the PCR products to replace either samR0478 or samR0479 in the BAC by homologous recombination. E. coli ET12567/pUZ8002 was separately transformed with

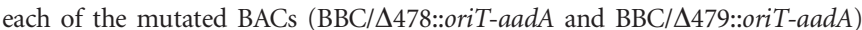
that were subsequently transferred independently to S. ambofaciens ATCC/ OE484 by conjugation. Spectinomycin-resistant colonies were picked and grown in Hickey-Tresner (HT) liquid medium. ${ }^{14}$ After several rounds of growth, cultures were spread on HT agar medium, and individual colonies were picked and analyzed by PCR and Southern hybridization to identify those in which the desired gene replacements had occurred via double crossover homologous recombination. The resulting mutants were named ATCC/OE484/ $\Delta 478$ and ATCC/OE484/ $\Delta 479$

\section{Incorporation of ${ }^{18} \mathrm{O}_{2}$ into stambomycins $\mathrm{A}-\mathrm{D}$ 1-4}

The ATCC/OE484 strain was cultured on MP5 agar medium overlaid with a sterile cellophane membrane as described previously ${ }^{12}$ in a glove bag filled with ${ }^{18} \mathrm{O}_{2}$ gas. After 4 days of incubation at $30^{\circ} \mathrm{C}$, the cellophane membrane was lifted off the plate and the mycelia were scraped into a $50 \mathrm{ml}$ plastic tube. Then, $10 \mathrm{ml}$ of methanol was added and the mixture was sonicated for $10 \mathrm{~min}$. The mycelia were pelleted by centrifugation for $10 \mathrm{~min}$ at $4{ }^{\circ} \mathrm{C}$ and the supernatant was analyzed by HR LC-MS using a flow rate of $0.2 \mathrm{ml} \mathrm{min}^{-1}$ and water containing $0.1 \%$ formic acid (solvent $\mathrm{A}$ )/acetonitrile containing $0.1 \%$ formic acid (solvent B) as eluents. The following elution profile was used: $80 \%$ solvent A $/ 20 \%$ solvent B for $5 \mathrm{~min}$; gradient to $100 \%$ solvent B in $15 \mathrm{~min} ; 100 \%$ solvent B for 5 min; gradient back to $80 \%$ solvent A/20\% solvent B in 3 min. The MS was calibrated at the beginning of each run via loop injection of $20 \mu \mathrm{l}$ of $10 \mathrm{~mm}$ sodium formate and was operated in positive ion mode with settings as follows. Full MS scan range: $50-2000 \mathrm{~m} / z$; End plate offset: $-500 \mathrm{~V}$; 
Table 1 Strains, plasmids and bacterial artificial chromosomes (BACs) used in this work

\begin{tabular}{|c|c|c|}
\hline Strain, BAC or plasmid & Principal characteristics ${ }^{\mathrm{a}}$ & Reference \\
\hline \multicolumn{3}{|l|}{ Strains: } \\
\hline \multicolumn{3}{|c|}{ Streptomyces ambofaciens } \\
\hline ATCC23877 & Wild type & 16 \\
\hline ATCC/OE484 & Second copy of samRO484 under the control of the $\mathrm{ermE}^{*}$ promoter & 12 \\
\hline ATCC/OE484/ 4478 & $\begin{array}{l}\text { Second copy of samRO484 under the control of the } e r m E^{*} \text { promoter and samRO478 replaced } \\
\text { by a spectinomycin resistance cassette }\end{array}$ & This work \\
\hline ATCC/OE484/ 4479 & $\begin{array}{l}\text { Second copy of samRO484 under the control of the } e r m E^{*} \text { promoter and samRO479 replaced } \\
\text { by a spectinomycin resistance cassette }\end{array}$ & This work \\
\hline \multicolumn{3}{|l|}{ Escherichia coli } \\
\hline $\mathrm{DH} 5 \alpha$ & General cloning strain & 17 \\
\hline ET12567/pUZ8002 & Nonmethylating strain used for conjugal transfer of mutated BACs to $S$. ambofaciens & 18 \\
\hline BW25113/pKD20 & Strain used for PCR-targeting mutagenesis (gam, bet, exo, bla) & 19 \\
\hline \multicolumn{3}{|l|}{ BAC or plasmid: } \\
\hline $\mathrm{BBC}$ & BAC from the genomic library of $S$. ambofaciens (cat) & 20 \\
\hline BBC/ $\Delta 478::$ oriT-aadA & samR0478 replaced by the spectinomycin resistance cassette in BBC (cat, aadA) & This work \\
\hline BBC/ $\triangle 479::$ oriT-aadA & samR0479 replaced by the spectinomycin resistance cassette in BBC (cat, aadA) & This work \\
\hline plJ778 & Contains the oriT-aadA spectinomycin resistance cassette & 13 \\
\hline
\end{tabular}

abla, ampicillin resistance gene; oriT, origin of transfer; aadA, spectinomycin/streptomycin resistance gene; gam, inhibitor of the host exonuclease V; bet, single-stranded DNA binding protein; exo, exonuclease promoting recombination along with bet; cat, chloramphenicol resistance gene.

capillary: $-4000 \mathrm{~V}$; nebulizer gas $\left(\mathrm{N}_{2}\right)$ : 1.6 bar; dry gas $\left(\mathrm{N}_{2}\right): 81 \mathrm{~min}^{-1}$; dry temperature: $180^{\circ} \mathrm{C}$.

\section{Analysis of the production of stambomycins and related metabolites in S. ambofaciens ATCC/OE484/ 4478 and ATCC/OE484/ $\triangle 479$}

S. ambofaciens ATCC/OE484/ 4478 was grown and analyzed as described above for the incorporation of ${ }^{18} \mathrm{O}_{2}$ into the stambomycins. S. ambofaciens ATCC/ OE484/ $\Delta 479$ was grown in liquid MP5 medium, as described previously. ${ }^{12}$ The culture supernatant was separated from the biomass by centrifugation and passed through a $0.4 \mu \mathrm{m}$ filter. The filtrate was analyzed by LC-MS, as described above for the incorporation of ${ }^{18} \mathrm{O}_{2}$ into the stambomycins.

\section{Isolation of 28-deoxystambomycins A-D 5-8 from S. ambofaciens ATCC/OE484/ $\triangle 478$}

The 28-deoxystambomycins A-D 5-8 were isolated from cultures of S. ambofaciens ATCC/OE484/ $\triangle 478$ grown on solid MP5 medium, as described previously for the isolation of stambomycins A-D 1-4 from S. ambofaciens ATCC/OE484. ${ }^{12}$

\section{Isolation of shunt metabolites 9-12 from S. ambofaciens ATCC/ OE484/ $\triangle 479$}

Twenty $50 \mathrm{ml}$ cultures of $S$. ambofaciens ATCC/OE484/ $\Delta 479$ were grown in MP5 liquid medium, as described previously. ${ }^{12}$ The cultures were combined and centrifuged for $20 \mathrm{~min}$ at 4000 r.p.m. $(3220 \mathrm{~g})$. The supernatant was freezedried and the residue was re-suspended in $25 \mathrm{ml}$ of 1:4 MeOH/ $\mathrm{H}_{2} \mathrm{O}$. The resulting solution was passed through a $0.4 \mu \mathrm{m}$ syringe filter and separated by HPLC, monitoring absorbance at $240 \mathrm{~nm}$. The column was eluted with water containing $0.1 \%$ formic acid (A) and acetonitrile containing $0.1 \%$ formic acid (B) using the following elution profile: $80 \%$ solvent $\mathrm{A} / 20 \%$ solvent $\mathrm{B}$ for $10 \mathrm{~min}$; gradient to $100 \%$ solvent B in $10 \mathrm{~min}$; $100 \%$ solvent B for $15 \mathrm{~min}$. Fractions containing 9-12 were identified using ESI-MS, combined and evaporated under reduced pressure. The residue was resuspended in $5 \mathrm{ml}$ of $50 \%$ aqueous methanol and further purified by HPLC using the following elution conditions: 0 min, $50 \% \mathrm{~A} / 50 \% \mathrm{~B} ; 15 \mathrm{~min}, 5 \% \mathrm{~A} / 95 \% \mathrm{~B} ; 20 \mathrm{~min}, 100 \%$ B; $25 \mathrm{~min}, 100 \%$ B. Fractions containing an inseparable mixture of 9 and $\mathbf{1 0}$ were collected, combined and lyophilized, as were fractions containing an inseparable mixture of $\mathbf{1 1}$ and $\mathbf{1 2}$, yielding 4.3 and $3.2 \mathrm{mg}$, respectively.

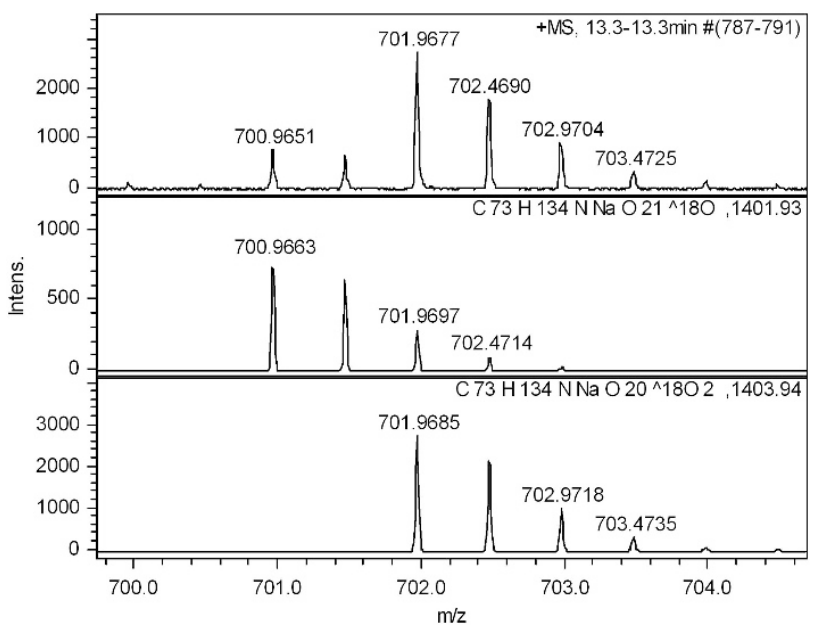

Figure 3 Mass spectra from LC-MS analysis of the incorporation of ${ }^{18} \mathrm{O}_{2}$ into stambomycins $A / B \quad \mathbf{1 / 2}$. The top panel shows the mass spectrum of a mixture of singly and doubly ${ }^{18}$ O-labeled stambomycins $A / B$; none of the unlabeled metabolites are observed. The middle panel shows the simulated mass spectrum for a species with the molecular formula $\mathrm{C}_{73} \mathrm{H}_{134} \mathrm{~N}^{16} \mathrm{O}_{21}{ }^{18} \mathrm{ONa}^{2+}$, corresponding to the doubly charged ion of singly labeled stambomycins $A / B$. The bottom panel shows the simulated mass spectrum for a species with the molecular formula $\mathrm{C}_{73} \mathrm{H}_{134} \mathrm{~N}^{16} \mathrm{O}_{20}{ }^{18}$ $\mathrm{O}_{2} \mathrm{Na}^{2+}$, corresponding to the doubly charged ion of doubly labeled stambomycins $\mathrm{A} / \mathrm{B}$.

\section{RESULTS}

If our hypothesis for the origin of the C-28 and C-50 oxygen atoms is correct, we would expect two oxygen atoms of the stambomycins to be derived from molecular oxygen. Thus, we investigated the incorporation of ${ }^{18} \mathrm{O}_{2}$ into stambomycins A-D 1-4. The previously reported stambomycin producer S. ambofaciens ATCC/OE484 ${ }^{12}$ was grown on a solid medium under an ${ }^{18} \mathrm{O}_{2}$ atmosphere and methanol extracts of the mycelia were analyzed by HR LC-MS. An approximately 3:1 mixture of doubly and singly ${ }^{18} \mathrm{O}$-labeled stambomycins A and B 1 and 2 was observed (Figure 3). Analogous 
${ }^{18} \mathrm{O}_{2}$ incorporation patterns were observed for stambomycins $\mathrm{C}$ and D 3 and 4.

The samR0478 and samR0479 genes within the stambomycin biosynthetic gene cluster encode proteins with sequence similarity to CYPs (Figure 4a). ${ }^{12}$ Thus, we hypothesized that SamR0478 and SamR0479 catalyze the hydroxylation of C-28 and C-50 (or vice versa) of the stambomycin polyketide chain. To investigate the role played by these enzymes in stambomycin biosynthesis, we independently replaced samR0478 and samR0479 on the chromosome of S. ambofaciens ATCC/OE484 with a spectinomycin resistance cassette.

HR LC-MS analysis of methanol extracts of the samR0478 mutant showed that the production of stambomycins A-D 1-4 was abolished and identified new metabolites with molecular formulae corresponding to stambomycin derivatives lacking an oxygen atom (calculated for $\mathrm{C}_{73} \mathrm{H}_{134} \mathrm{NO}_{21} \mathrm{Na}^{2+}$ : 691.9668, found: 691.9682; calculated for $\mathrm{C}_{72} \mathrm{H}_{132} \mathrm{NO}_{21} \mathrm{Na}^{2+}$ : 684.9589, found: 684.9601) (Supplementary Information). The new metabolites were purified from extracts of large-scale cultures of the samR0478 mutant by semipreparative HPLC. ${ }^{1} \mathrm{H}$ and ${ }^{13} \mathrm{C}$ NMR data for the purified metabolites were very similar to those for stambomycins A-D 1-4 (Supplementary Information). Only the signals due to $\mathrm{C}-28$ differed significantly $\left(\delta_{\mathrm{H}}=3.47\right.$ p.p.m. and $\delta_{\mathrm{C}}=74.2$ p.p.m. for stambomycins $\mathrm{A} / \mathrm{B}$; $\delta_{\mathrm{H}}=1.30,1.58$ p.p.m. and $\delta_{\mathrm{C}}=35.8$ p.p.m. for the corresponding novel metabolites), leading us to conclude that the novel metabolites are 28-deoxystambomycins A-D 5-8 (Figure 4b and Supplementary Information).

The production of stambomycins A-D 1-4 was also found to be abolished in the samR0479 mutant, which accumulated new metabolites with the molecular formulae $\mathrm{C}_{65} \mathrm{H}_{120} \mathrm{O}_{19}$ (calculated for $[\mathrm{M}+$ $\mathrm{H}]^{+}$: 1205.8497, found: 1205.8488 ) and $\mathrm{C}_{64} \mathrm{H}_{118} \mathrm{O}_{19}$ (calculated for $[\mathrm{M}+\mathrm{H}]^{+}$: 1191.8340, found: 1191.8332) (Supplementary Information). A neutral loss of $44 \mathrm{Da}$, corresponding to $\mathrm{CO}_{2}$, was observed in negative ion mode MS/MS analyses of the purified metabolites (Supplementary Information). This indicated that they are the shunt metabolites $\mathbf{9 - 1 2}$, resulting from hydrolytic release and

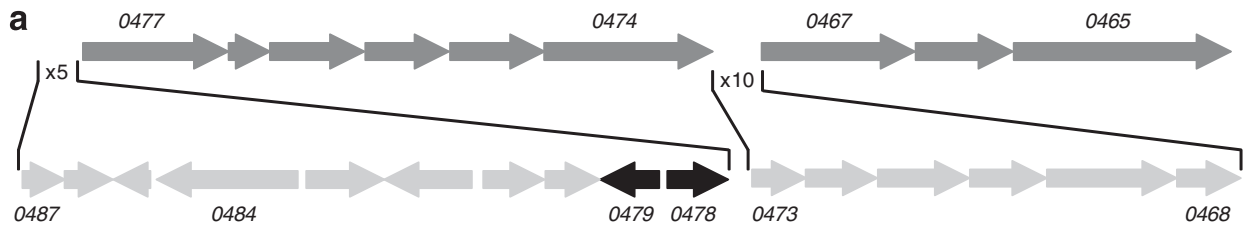

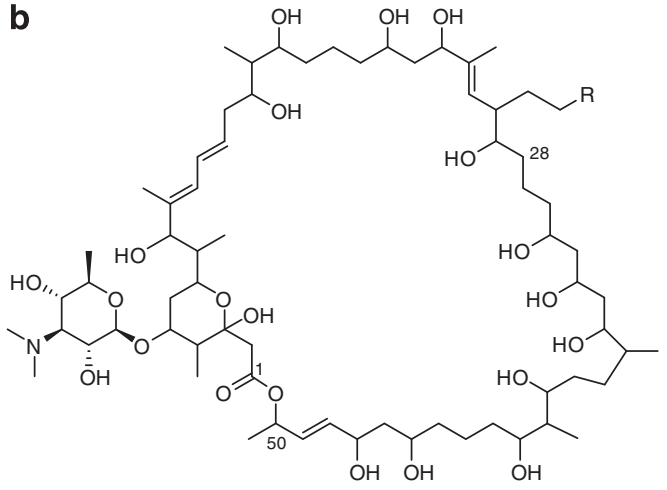

28-deoxystambomycin A $5\left(\mathrm{R}=\mathrm{CH}_{2} \mathrm{CH}\left(\mathrm{CH}_{3}\right) \mathrm{CH}_{2} \mathrm{CH}_{3}\right)$ 28-deoxystambomycin B $6\left(\mathrm{R}=\mathrm{CH}_{2} \mathrm{CH}_{2} \mathrm{CH}\left(\mathrm{CH}_{3}\right) \mathrm{CH}_{3}\right)$ 28-deoxystambomycin $\mathrm{C} 7\left(\mathrm{R}=\mathrm{CH}_{2} \mathrm{CH}\left(\mathrm{CH}_{3}\right) \mathrm{CH}_{3}\right)$ 28-deoxystambomycin D $8\left(\mathrm{R}=\mathrm{CH}_{2} \mathrm{CH}_{2} \mathrm{CH}_{2} \mathrm{CH}_{3}\right)$

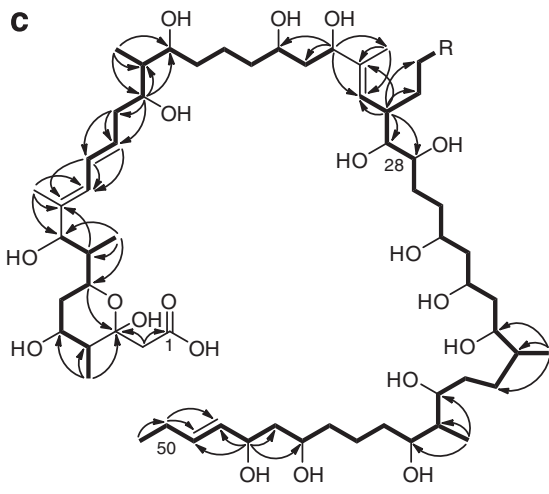

$$
\begin{aligned}
& 9\left(\mathrm{R}=\mathrm{CH}_{2} \mathrm{CH}\left(\mathrm{CH}_{3}\right) \mathrm{CH}_{2} \mathrm{CH}_{3}\right) \\
& 10\left(\mathrm{R}=\mathrm{CH}_{2} \mathrm{CH}_{2} \mathrm{CH}(\mathrm{CH}) \mathrm{CH}_{3}\right) \\
& 11\left(\mathrm{R}=\mathrm{CH}_{2} \mathrm{CH}\left(\mathrm{CH}_{3}\right) \mathrm{CH}_{3}\right) \\
& 12\left(\mathrm{R}=\mathrm{CH}_{2} \mathrm{CH}_{2} \mathrm{CH}_{2} \mathrm{CH}_{3}\right)
\end{aligned}
$$

Figure 4 The stambomycin biosynthetic gene cluster and the structures of stambomycin-related metabolites accumulated in the samR0478 and samR0479 mutants of Streptomyces ambofaciens. (a) Organization of the stambomycin biosynthetic gene cluster. The samR0478 and samR0479 genes, which were independently deleted in this study, are black. The polyketide synthase (PKS) genes are dark gray and other genes are light gray. The samR0487 to samR0478 and samR0473-samR0468 regions are magnified 5 and 10 times, respectively, compared with the regions containing the PKS genes. (b) Structures of 28-deoxystambomycins A-D 5-8 accumulated in the samR0478 mutant. (c) Structures of the shunt metabolites 9-12 that accumulate in the samR0479 mutant showing observed COSY correlations (black lines) and key heteronuclear multiple-bond correlations (HMBC; arrows).

Figure 5 Proposed roles of the putative cytochromes $P 450$ (CYPs) encoded by samR0478 and samR0479 in stambomycin biosynthesis, and mechanisms

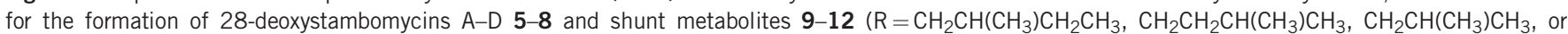
$\mathrm{CH}_{2} \mathrm{CH}_{2} \mathrm{CH}_{2} \mathrm{CH}_{3}$ ) in the samR0478 and samR0479 mutants, respectively, of Streptomyces ambofaciens. SamR0478 and SamR0479 are hypothesized to catalyze hydroxylation of $\mathrm{C}-28$ and $\mathrm{C}-50$, respectively, of the fully assembled polyketide chains attached to the acyl carrier protein (ACP) domain within the last polyketide synthase (PKS) module. Alternatively, these enzymes may catalyze hydroxylation of earlier intermediates in polyketide chain assembly. In the samR0478 mutant, the ACP-bound intermediates in which $\mathrm{X}=\mathrm{H}$ and $\mathrm{Y}=\mathrm{OH}$ are transferred to the active site Ser residue of the thioesterase (TE) domain that catalyzes chain release via macrolactonization utilizing the C-50 hydroxyl group introduced by SamR0479. The resulting $28-$ deoxystambomycin A-D algycones 13-16 undergo SamR0481-mediated mycaminosylation, yielding 28-deoxystambomycins A-D 5-8. In contrast, the ACP-bound intermediates in which $\mathrm{X}=\mathrm{OH}$ and $\mathrm{Y}=\mathrm{H}$ accumulate in the samR0479 mutant. These intermediates are transferred to the active site Ser residue of the TE domain, but cannot be offloaded via macrolactonization because the C-50 hydroxyl group is absent. Instead, the TE domain catalyzes hydrolysis of the enzyme-bound intermediates, yielding shunt metabolites 9-12. 


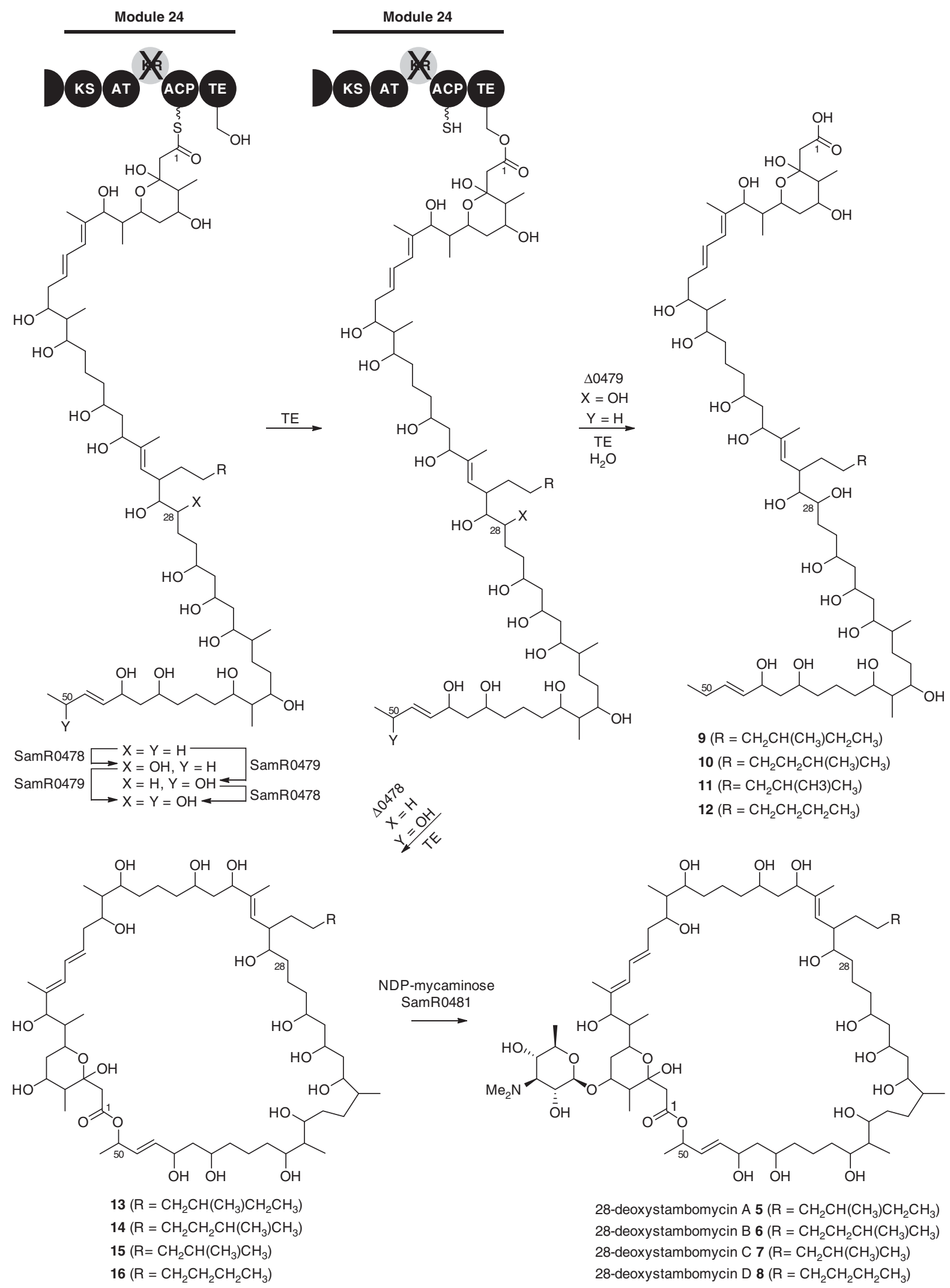


C-28 hydroxylation of the fully assembled polyketide chains from the stambomycin PKS. ${ }^{1} \mathrm{H},{ }^{13} \mathrm{C}$, COSY, TOCSY, heteronuclear single quantum correlation and heteronuclear multiple-bond correlation NMR spectroscopic analyses confirmed this hypothesis and showed that these metabolites possess the C-28 hydroxyl group, but lack the C-50 hydroxyl group required for macrolide formation (Figure $4 \mathrm{c}$ and Supplementary Information). Coupling constants of 15.0 and $15.2 \mathrm{~Hz}$ for $\mathrm{H}-12 / \mathrm{H}-13$ and $\mathrm{H}-48 / \mathrm{H}-49$, respectively, confirmed the $E$ configurations of the C-12/C-13 and C-48/C-49 double bonds in 9 and $\mathbf{1 0}$. Similarly, correlations between the C-10 methyl group and $\mathrm{H}-12$, and the C-24 methyl group and H-26 in the NOESY spectrum of 9 and 10, were consistent with $E$ configurations for the C-10/C-11 and C-24/C-25 double bonds (Supplementary Information).

\section{DISCUSSION}

The incorporation of two atoms of oxygen from ${ }^{18} \mathrm{O}_{2}$ into stambomycins A-D 1-4 is consistent with our hypothesis that the C-28 and C-50 oxygen atoms of these metabolites derive from hydroxylation of the polyketide chain. The accumulation of 28-deoxystambomycins A-D 5-8 in the samR0478 mutant of S. ambofaciens implies that the putative CYP encoded by samR0478 is responsible for introducing the C-28 hydroxyl group into the stambomycins. Whether this hydroxylation reaction occurs during or after polyketide chain assembly is unclear. The observation that the shunt metabolites accumulated in the samR0479 mutant have a hydroxyl group at C-28 tempts us to speculate that C-28 hydroxylation occurs during polyketide chain assembly (Figure 5). Several examples of analogous CYP-catalyzed in trans oxidations have been reported for intermediates bound to the carrier proteins of nonribosomal peptide synthetases. ${ }^{15}$

The accumulation of stambomycin shunt metabolites 9-12, lacking the C-50 oxygen atom of the macrolide and the mycaminose residue, in the samR0479 mutant indicates that the putative CYP encoded by samR0479 catalyzes hydroxylation of C-50 during (or possibly even before) polyketide chain assembly (Figure 5). It appears that the TE domain of the PKS is unable to catalyze chain release via macrolactonization if the C-50 hydroxyl group is absent from the polyketide chains, despite the availability of several alternative hydroxyl groups resulting from keto reduction during chain assembly. Thus, the fully assembled polyketide chains appear to be offloaded from the PKS in the samR0479 mutant via hydrolysis (Figure 5). In contrast to the 28-deoxystambomycin A-D aglycones 13-16, 9-12 are evidently not substrates for the mycaminosyl transferase encoded by samR0481 12 (Figure 5).

In conclusion, we have shown that putative CYPs encoded by the samR0478 and samR0479 genes are involved in the introduction of the $\mathrm{C}-28$ hydroxyl group and the $\mathrm{C}-50$ oxygen atom, respectively, into the stambomycins. Hydroxylation at C-50 is required for offloading of fully assembled polyketide chains from the stambomycin PKS via macrolactonization. Further insight into the roles played by SamR0478 and SamR0479 in stambomycin biosynthesis could be obtained by investigating the catalytic properties of the purified recombinant proteins. However, this may prove challenging, given the structural complexity of likely substrates for these enzymes.

\section{ACKNOWLEDGEMENTS}

This research was supported by the European Commission under the 6th framework program (integrated project Actinogen; FP6-5224).The Bruker MaXis mass spectrometer used in this research was obtained through Birmingham Science City: Innovative Uses for Advanced Materials in the Modern World (West Midlands Centre for Advanced Materials Project 2), with support from Advantage West Midlands and was partly funded by the European Regional Development Fund.

1 Harris, D. R., McGeachin, S. G. \& Mills, H. H. The structure and stereochemistry of erythromycin A. Tetrahedron Lett. 679-685 (1965).

2 Ganis, P., Avitabile, G., Mechlinski, W. \& Schaffner, C. P. Polyene macrolide antibiotic amphotericin B. Crystal structure of the $\mathrm{N}$-iodoacetyl derivative. J. Am. Chem. Soc. 93, 4560-4564 (1971).

3 Chong, C. N. \& Rickards, R. W. Macrolide antibiotic studies. XVI. The structure of nystatin. Tetrahedron Lett. 5145-5148 (1970).

4 Springer, J. P., Arison, B. H., Hirshfield, J. M. \& Hoogsteen, K. The absolute stereochemistry and conformation of avermectin B2a aglycone and avermectin Bla. J. Am. Chem. Soc. 103, 4221-4224 (1981).

5 Kirst, H. A. et al. A83543A-D, unique fermentation derived tetracyclic macrolides. Tetrahedron Lett. 32, 4839-4842 (1991).

6 Donadio, S. \& Katz, L. Organization of the enzymatic domains in the multifunctional polyketide synthase involved in erythromycin formation in Saccharopolyspora erythraea. Gene 111, 51-60 (1992).

7 Caffrey, P., Lynch, S., Flood, E., Finnan, S. \& Oliynyk, M. Amphotericin biosynthesis in Streptomyces nodosus: deductions from analysis of polyketide synthase and late genes. Chem. Biol. 8, 713-723 (2001).

8 Brautaset, T. et al. Biosynthesis of the polyene antifungal antibiotic nystatin in Streptomyces noursei ATCC 11455: analysis of the gene cluster and deduction of the biosynthetic pathway. Chem. Biol. 7, 395-403 (2000).

9 Waldron, C. et al. Cloning and analysis of the spinosad biosynthetic gene cluster of Saccharopolyspora spinosa. Chem. Biol. 8, 487-499 (2001).

10 Ikeda, H., Nonomiya, T., Usami, M., Ohta, T. \& Omura, S. Organization of the biosynthetic gene cluster for the polyketide anthelmintic macrolide avermectin in Streptomyces avermitilis. Proc. Natl Acad. Sci. USA 96, 9509-9514 (1999).

11 Tsai, S.-C. et al. Crystal structure of the macrocycle-forming thioesterase domain of the erythromycin polyketide synthase: versatility from a unique substrate channel. Proc. Natl Acad. Sci. USA 98, 14808-14813 (2001).

12 Laureti, L. et al. Identification of a bioactive 51-membered macrolide complex by activation of a silent polyketide synthase in Streptomyces ambofaciens. Proc. Natl Acad. Sci. USA 108, 6258-6263 (2011).

13 Gust, B., Challis, G. L., Fowler, K., Kieser, T. \& Chater, K. F. PCR-targeted Streptomyces gene replacement identifies a protein domain needed for biosynthesis of the sesquiterpene soil odor geosmin. Proc. Natl Acad. Sci. USA 100, 1541-1546 (2003)

14 Kieser, T., Bibb, M. J., Buttner, M. J., Chater, K. F. \& Hopwood, D. A. Practical Streptomyces Genetics 407, (John Innes Foundation, Norwich, 2000).

15 Cryle, M. J. Carrier protein substrates in cytochrome P450-catalysed oxidation. Metallomics 3, 323-326 (2011).

16 Pinnert-Sindico, S. Une nouvelle espèce de Streptomyces productrice d'antibiotiques: Streptomyces ambofaciens n. sp. caractères culturaux. Ann. Inst. Pasteur. (Paris) 87, 702-707 (1954)

17 Hanahan, D. Studies on transformation of Escherichia coli with plasmids. J. Mol. Biol. 166, 557-580 (1983).

$18 \mathrm{MacNeil,} \mathrm{D.} \mathrm{J.} \mathrm{et} \mathrm{al.} \mathrm{Analysis} \mathrm{of} \mathrm{Streptomyces} \mathrm{avermitilis} \mathrm{genes} \mathrm{required} \mathrm{for} \mathrm{avermectin}$ biosynthesis utilizing a novel integration vector. Gene 111, 61-68 (1992).

19 Datsenko, K. A. \& Wanner, B. L. One-step inactivation of chromosomal genes in Escherichia coli K-12 using PCR products. Proc. Natl Acad. Sci. USA 97, 6640-6645 (2000)

20 Choulet, F. et al. Evolution of the terminal regions of the Streptomyces linear chromosome. Mol. Biol. Evol. 23, 2361-2369 (2006).

Supplementary Information accompanies the paper on The Journal of Antibiotics website (http://www.nature.com/ja) 